\title{
Three-dimensional computed tomography mapping of 136 tongue-type calcaneal fractures from a single centre
}

\author{
Xiaobo Guo ${ }^{1}$, Xiaonan Liang ${ }^{1}$, Jiangtao Jin ${ }^{2}$, Jinwei Chen ${ }^{2}$, Junyang Liu ${ }^{2}$, Yu Qiao ${ }^{1}$, Jianwen Cheng ${ }^{1}$, \\ Jinmin Zhao ${ }^{1}$ \\ ${ }^{1}$ Department of Trauma Orthopaedics and Hand Surgery, The First Affiliated Hospital of Guangxi Medical University, Nanning, China; \\ ${ }^{2}$ Department of Orthopaedics, Jincheng General Hospital, Jincheng, China \\ Contributions: (I) Conception and design: X Guo, J Cheng, J Zhao; (II) Administrative support: J Cheng, J Zhao; (III) Provision of study materials or \\ patients: X Guo, J Jin, J Chen, J Liu; (IV) Collection and assembly of data: X Guo, X Liang, Y Qiao; (V) Data analysis and interpretation: X Guo, X \\ Liang; (VI) Manuscript writing: All authors; (VII) Final approval of manuscript: All authors. \\ Correspondence to: Jianwen Cheng; Jinmin Zhao. Department of Trauma Orthopaedics and Hand Surgery, The First Affiliated Hospital of Guangxi \\ Medical University, Nanning 530021, China. Email: 1730054162@qq.com; csgkswk@126.com.
}

Background: This study aimed to evaluate tongue-type calcaneal fractures using three-dimensional (3D) computed tomography (CT) mapping method.

Methods: A consecutive series of 136 tongue-type calcaneal fractures was used. CT data were used to reconstruct and reposition the 3D calcaneus body. E-3D Medical 18.01 software was used to superimpose the fractured calcaneal entity on the template, and the fracture line was drawn on the template. Finally, the heatmap was obtained using the fracture statistical analysis function. At the same time, the distribution of fracture lines in the anterior part of the calcaneus (APC) was recorded. Cases were divided into the following 3 subtypes according to the distribution of the tongue-type fracture lines of the calcaneal body: mediallateral (Group A), upper-lateral (Group B), and upper-lateral-medial (Group C).

Results: There were 68 cases in Group A, 48 cases in Group B, and 20 cases in Group C. Based on subtype, the characteristic fracture map of Groups A, B and C was constructed. The APC was evenly divided into zones A, B, and C from lateral to medial. In Group A, the most common types involve APC were Type AC (24, 35.3\%) and Type A (11, 16.2\%). In Group B, the most common types were Type AC (20, 41.7\%), Type AB $(8,16.7 \%)$ and Type C $(8,16.7 \%)$. In Group C, the most common types were Type AC $(8,40.0 \%)$ and Type A $(5,25.0 \%)$.

Conclusions: This study investigates the distribution characteristics of fracture lines in subgroups of tongue-type calcaneal fractures for the first time. The results can help doctors improve their understanding of tongue-type calcaneal fractures, optimize internal fixation design, and provide a standard model for biomechanical experiments.

Keywords: Calcaneus; computed tomography (CT); three-dimensional (3D); intra-articular fractures; fracture fixation

Submitted Nov 05, 2021. Accepted for publication Dec 08, 2021.

doi: $10.21037 / \mathrm{atm}-21-6168$

View this article at: https://dx.doi.org/10.21037/atm-21-6168

\section{Introduction}

Calcaneus fracture is the most common fracture of the tarsal fractures. Epidemiological results show that calcaneal fractures account for $2 \%$ of all fractures and $60 \%$ of tarsal fractures (1). Calcaneal fractures are mostly caused by high- energy injuries. Falling from heights and traffic injuries account for the majority of fractures, with most patients being young and middle-aged. The main symptoms are soft tissue swelling, pain, deformity of the foot, and longterm foot discomfort and symptoms last for 3 to 5 years (2). 
Most calcaneal fractures are intra-articular fractures, which increase the width of the calcaneus, involve the subtalar joint surface, and reduce the Bohler angle. Improper treatment may lead to poor outcomes and disability $(3,4)$. In the treatment of calcaneal fractures, there is a high qualifying requirement for the reduction of the fracture fragments, and therefore, the treatment of calcaneal fractures is a challenge for orthopaedic surgeons.

There are multiple classifications of calcaneal fractures. Currently, the commonly used are Essex-Lopresti and Sanders classifications. According to the Essex-Lopresti classification, fractures are divided into tongue-type fracture and joint compression fracture (5). However, the EssexLopresti classification has limitations. It cannot assess damage to the subtalar joint and is of little significance for treatment and prognosis (6). With the development of imaging diagnosis technology, especially computed tomography (CT) technology, clinicians can analyze calcaneal fractures from a new perspective. The Sanders classification of calcaneal fractures has been proposed (7), which is very useful in predicting prognosis and intraoperative reduction; however, the distribution of fracture lines from the overall calcaneus is not described.

The fracture mapping was first proposed in 2009 by Armitage $e t$ al. (8). This technology uses medical threedimensional (3D) software to reconstruct 3D fracture entities. Then, multiple 3D entities of fracture cases are superimposed on the template through the registration function. The fracture lines are drawn out on the template one by one. Use the fracture statistical analysis function to generate a fracture line heatmap. The fracture heatmap can display the start and end, direction, and distribution of the fracture line in the form of big data, so as to help the doctor understand the distribution characteristics of fracture, guide the intraoperative reduction, and the design of the internal fixation device. Recently, fracture mapping has been used in clinical treatment of patella and distal humerus fractures $(9,10)$.

Our research group had previously conducted a fracture map study of Essex-Lopresti classification intra-articular compression calcaneal fractures (11). This study intends to use CT data of calcaneal fractures to reconstruct EssexLopresti classification tongue-type calcaneal fractures, draw the fracture line on the template, and generate a $3 \mathrm{D}$ heatmap of calcaneal fractures. We hypothesized that this study would demonstrate precise about the tongue-type fracture line distribution. This study may help doctors improve their understanding of tongue-type calcaneal fractures, optimize internal fixation design, and provide a standard model for biomechanical experiments. We present the following article in accordance with the MDAR reporting checklist (available at https://dx.doi.org/10.21037/ atm-21-6168).

\section{Methods}

\section{Patient cobort}

All procedures performed in this study involving human participants were in accordance with the Declaration of Helsinki (as revised in 2013). The study was approved by institutional ethics board of Jincheng General Hospital (No. 20200901) and informed consent was taken from all the patients. A total of 136 tongue-type calcaneal fractures in Jincheng General Hospital from 2017 to 2021 were collected. The inclusion criteria were as follows: (I) age: 18-70 years; (II) CT data of the calcaneus obtained preoperatively; (III) tongue-type calcaneus fracture; and (IV) closed calcaneal fractures. The exclusion criteria were as follows: (I) pathological fractures; (II) calcaneus deformity; (III) comminuted fracture with unidentifiable fracture line; (IV) past ankle fractures and ankle arthritis; (V) osteoporosis; and (VI) open fractures.

\section{Obtaining the calcaneal template}

To obtain the calcaneal template, a 30-year-old man (who signed the informed consent) with no previous systemic disease or foot and ankle trauma, arthritis, or deformity had both ankles and feet scanned using a 64-slice spiral CT (Siemens, Germany) with a thickness of $0.75 \mathrm{~mm}$. The CT data were obtained in the Digital Imaging and Communications in Medicine (DICOM) format. Next, the $3 \mathrm{D}$ entity of the right calcaneus was extracted using Mimics Research 20.0 software (Materialise, Leuven, Belgium). Using the Geomagic Studio 2014 (Geomagic, USA) software, the following operations were performed: remeshing of the 3D solid, removal of the spike, appropriate smoothing to generate a $3 \mathrm{D}$ solid model of the calcaneus, and importing it into the E-3D Medical 18.01 (Central South University, Changsha, China) as a template.

\section{Obtaining CT data and $3 D$ reconstruction of calcaneal fractures}

All cases were scanned using 64-slice spiral CT (Siemens, Germany). The scanning parameters were as follows: 

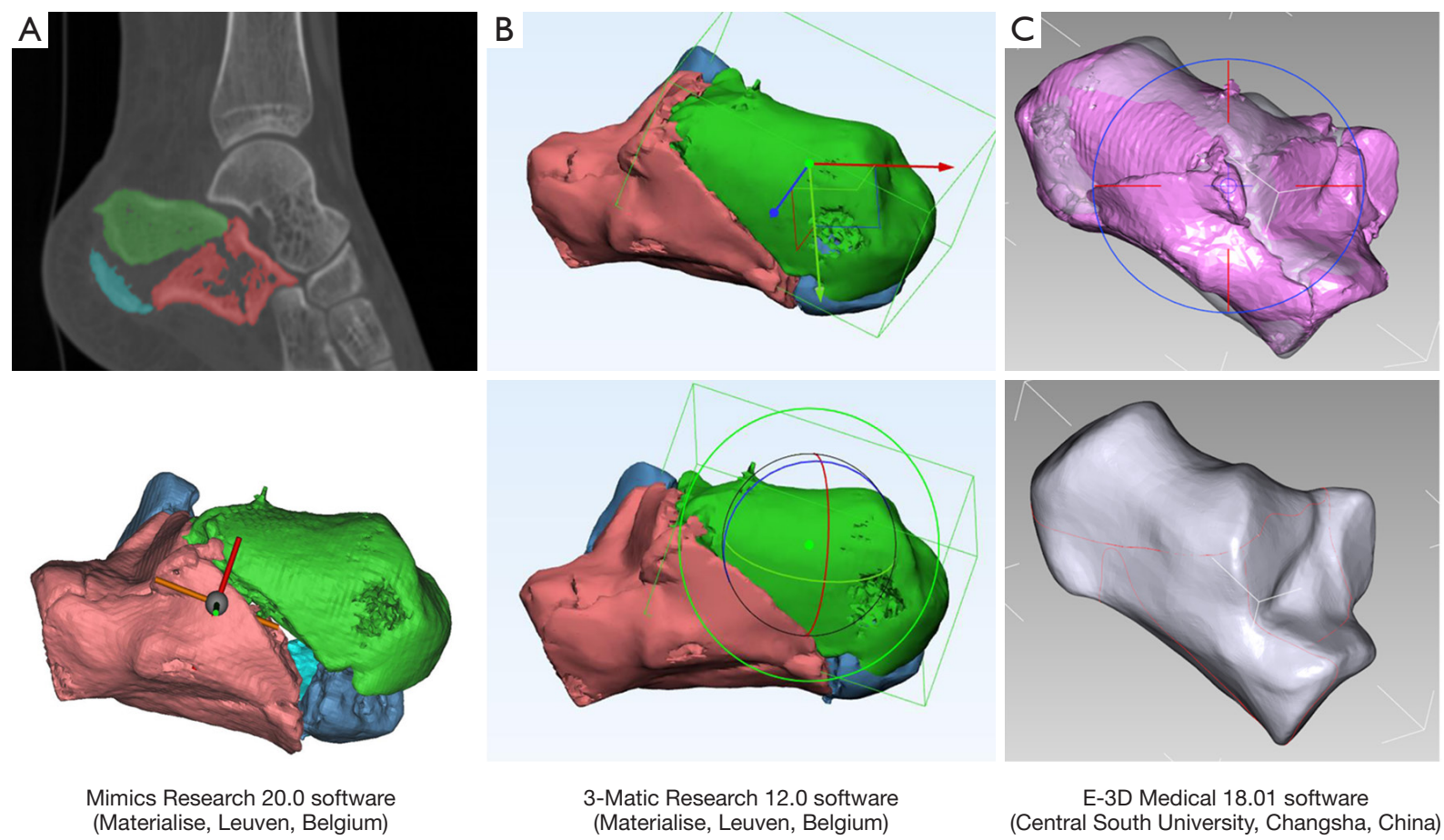

Mimics Research 20.0 software (Materialise, Leuven, Belgium)

(Materialise, Leuven, Belgium)

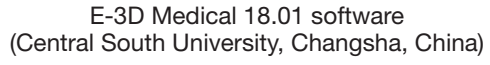

Figure 1 Flow charts of the calcaneal fracture map processing. (A) Using Mimics Research 20.0 software, the segment and split functions were applied to create each segment of calcaneal fracture 3D entity; (B) using 3-Matic Research 12.0 software to reposition each calcaneal fracture fragment; (C) after superimposing the fractured calcaneus entity with calcaneus template, the fracture line was drawn on the template using E-3D Medical 18.01 software. 3D, three-dimensional.

$120 \mathrm{kV}, 350 \mathrm{~mA}$, and layer thickness $<1.5 \mathrm{~mm}$. The scan range was from the ankle to the sole. CT data was saved in the DICOM format. The obtained CT data of calcaneal fractures were imported into Mimics Research 20.0 software (Materialise, Leuven, Belgium). The segmentation command was then used to extract the bone of the ankle, and the split command was used to segment the calcaneus and the other ankle bones. If the fracture fragments were significantly displaced, the split command was used again to segment each fragment such that each fragment had independent attributes. The fracture cases was imported into the 3-Matic Research 13.0 software (Materialise, Leuven, Belgium) as 3D entity. The 3D entity was reset by rotation and translation if necessary. Then, import the 3D entity into the E-3D Medical 18.01 (Central South University, Changsha, China) software and make it coincide with the template through registration, rotation, and translation functions. All cases performed the same operation. In the fracture line heatmap analysis, the area within $2 \mathrm{~mm}$ of the fracture line was defined as the fracture line area. The closer the fracture line is, the higher its weighting. By overlapping the fracture lines of all patients, the heatmap of fracture lines in different regions on the $3 \mathrm{D}$ model was calculated (Figure 1).

\section{Fracture subtype analysis and data collection}

The body of the calcaneus is divided into upper, medial, and lateral surfaces. The position of the tongue-type fragments fracture lines of the body were recorded and divided into the following subtypes: medial-lateral (Group A), upperlateral (Group B), and upper-lateral-medial (Group C). The number in each subtype was recorded, and the fracture heatmap was drawn to display its characteristics. According to our previous research, the anterior part of the calcaneus (APC) was evenly divided into zones A, B, and C from lateral to medial, and the zone affected by the main fracture line of each subtype was recorded (11) (Figure 2). 
Table 1 Patient demographics and subtypes of tongue-type calcaneal fracture $(\mathrm{n}=136)$

\begin{tabular}{lc}
\hline Characteristics & Value \\
\hline Age, mean \pm SD & $45.83 \pm 11.72$ \\
Sex, $\mathrm{n}(\%)$ & $132(97.06)$ \\
Male & $4(2.94)$ \\
Female & \\
Side of injury, $\mathrm{n}(\%)$ & $77(56.62)$ \\
Left & $59(43.38)$ \\
Right & \\
Subtypes, $\mathrm{n}(\%)$ & $68(50.00)$ \\
Group A & $48(35.29)$ \\
Group B & $20(14.71)$ \\
Group C & \\
Injury patterns, $\mathrm{n}(\%)$ & $103(75.74)$ \\
Falling from height & $29(21.32)$ \\
Falling from stairs & $4(2.94)$ \\
Car accident & \\
\hline
\end{tabular}

$\mathrm{SD}$, standard deviation.

\section{Statistical analysis}

This study used Statistical Product and Service Solutions (SPSS) software v.26.0 (IBM Inc., Armonk, NY, USA) for data processing. Continuous data were expressed as the means \pm standard deviation (SD), and quantitative data were expressed as frequencies and percentages. All cases were divided into subtypes Group A, Group B, and Group C. The chi-square or Fisher's exact test was used to analyze the differences between the subtypes, and $\mathrm{P}<0.05$ was considered statistically significant.

\section{Results}

\section{Demographic data}

From January 2017 to December 2021, we collected the CT data of 136 cases of tongue-type calcaneal fracture, including 132 (97.06\%) males and 4 (2.94\%) females [aged $18-70(45.83 \pm 11.72)$ years]. The causes of injury were as follows: falling from a height (103, $75.74 \%)$, falling downstairs $(29,21.32 \%)$, and car accident (4, 2.94\%). A total of 77 cases $(56.62 \%)$ involved the left calcaneus, and $59(43.38 \%)$ cases involved the right calcaneus (Table 1).
Features of the medial-lateral fracture line of the calcaneal body (Group A)

There were $68(50.00 \%)$ patients in Group A. The concentrated area of the transverse fracture line was located in the lower part of the posterior subtalar articular surface. The fracture line ran medially, partly through the middle and posterior part of the subtalar articular surface, and partly through the middle and posterior subtalar articular surface gap. At the root of the sustentaculum tali, the two parts of the fracture line were fused and ran backward and downward, and then divided into two branches: a branch that ran on the plantar surface anterior to the calcaneal tuberosity, and another that ran horizontally in the middle of the calcaneal tuberosity. The transverse fracture line on the subtalar articular surface ran laterally at the angle of Gissane and was divided into two branches: a branch that ran obliquely backward and downward, and merged with the fracture line anterior to the calcaneal tuberosity, and another that ran posteriorly and merged with the fracture line of the middle part of the calcaneal tuberosity. The fracture line concentrated area of APC was the lateral wall and the transition area between the anterior calcaneus and the sustentaculum tali (Figures 3,4).

\section{Features of the upper-lateral fracture line of the calcaneal body (Group B)}

There were 48 (35.29\%) patients in Group B. The concentrated area of the longitudinal fracture line was located in the middle part of the posterior subtalar articular surface and ran longitudinally on the upper side of the calcaneal body to the back, and then divided into two branches posteriorly and medially. The medial fracture line ran anterior to the calcaneal tuberosity, with a part that ran on the plantar surface and a part that ran longitudinally and anteriorly. The posterior fracture line was arc-shaped on the upper part of the calcaneal tuberosity. The longitudinal fracture line of the subtalar articular joint was divided into three branches anteriorly, which ran on the lateral wall of the calcaneus as well as the medial and lateral sides of the anterior calcaneus. On the lateral side, the fracture line was divided into two branches at the Gissane angle. One branch ran posteriorly and confluenced with the fracture line of the calcaneal tubercle to form a tongue fracture fragment, while the other branch ran obliquely in a posterior direction at the sole of the foot and confluenced with the anterior 
A
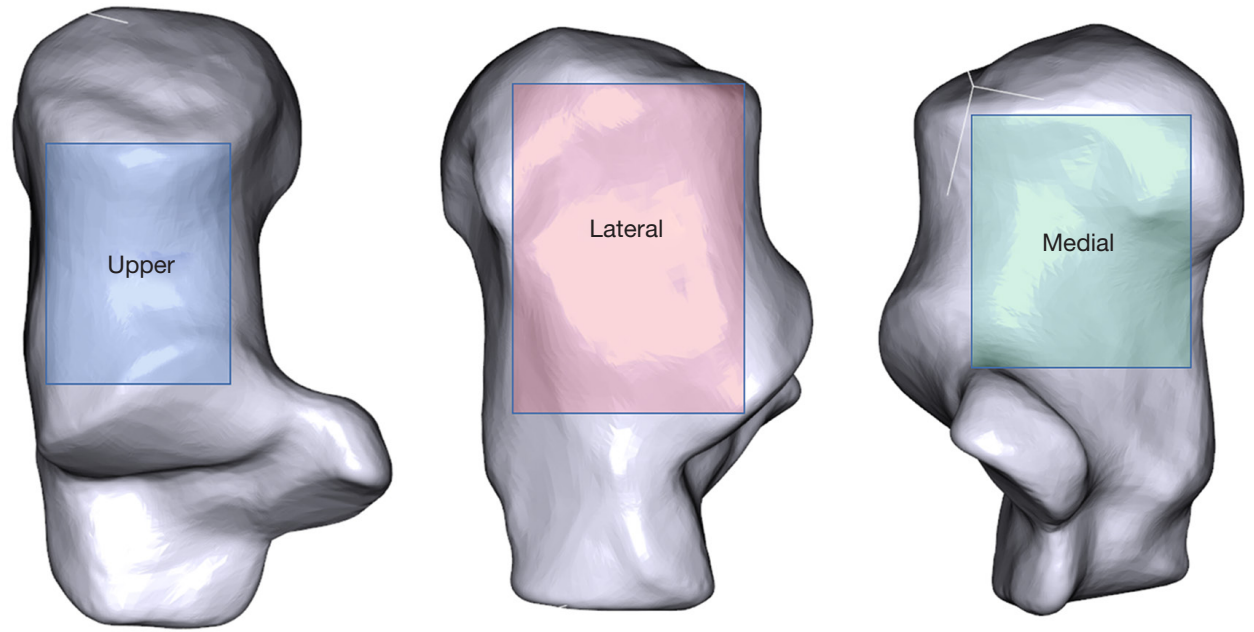

B
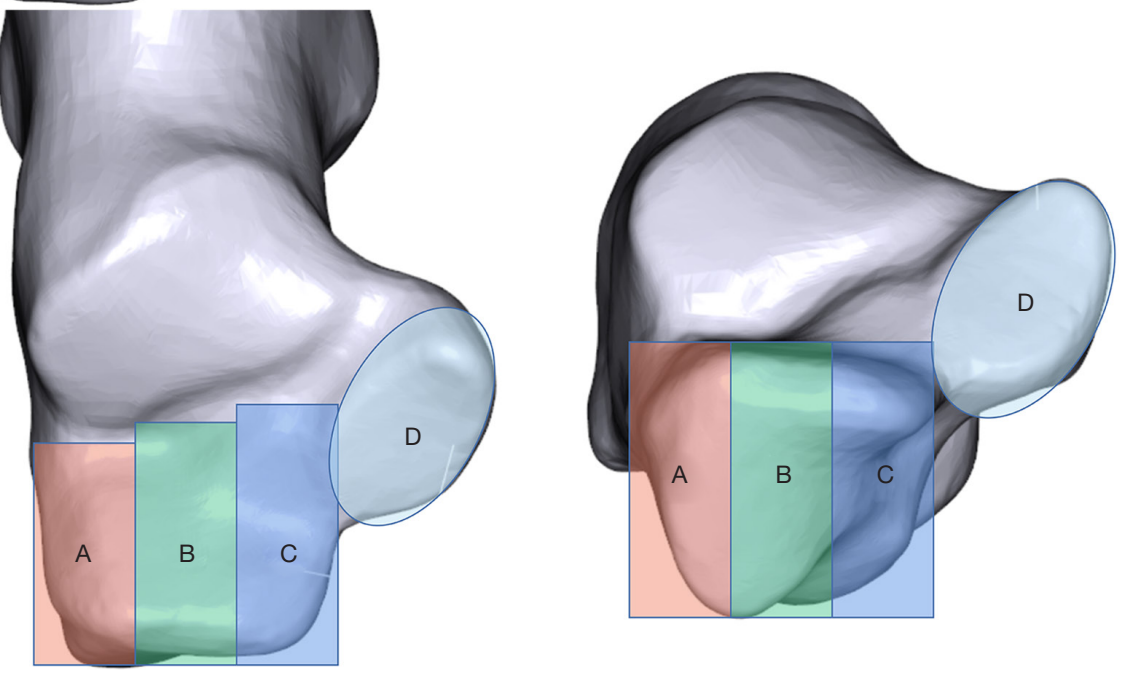

Figure 2 The division of the calcaneal body and the APC. (A) The calcaneal body was divided into upper, lateral and medial surface; (B) the APC was evenly divided into zones A, B, and C from lateral to medial, created by Guo et al. (11). APC, anterior part of the calcaneus.

line of the calcaneal tuberosity. The fracture lines of APC were concentrated in the lateral wall and the transitional area between the anterior calcaneus and sustentaculum tali (Figures 5,6).

\section{Features of the upper lateral-medial fracture line of the calcaneal body (Group C)}

There were 20 (14.71\%) patients in Group C. The fracture line of the posterior subtalar joint had both vertical and horizontal features. The vertical fracture line extended to the dorsal side of the calcaneal body and merged with the longitudinal fracture line of the lateral surface of the calcaneus. The horizontal fracture line extended to the medial side of the calcaneus through the sustentaculum tali.
This fracture line continued to pass through the anterior aspect of the calcaneal tuberosity and merged with the oblique fracture line on the lateral side of the calcaneus. The fracture lines of APC were concentrated in the lateral wall and the transitional area of anterior calcaneus and sustentaculum tali (Figures 7,8).

\section{Fracture distribution in the APC area.}

The Group A fracture line involved the APC and is represented as zone A $(53,77.9 \%)$, zone B $(18,26.5 \%)$, zone C (34, 50.0\%), and no involvement (14, 20.6\%). The Group B fracture line involved the anterior calcaneus and is represented as zone A $(32,66.7 \%)$, zone $\mathrm{B}(16,33.3 \%)$, zone $\mathrm{C}(32,66.7 \%)$, and no involvement $(1,2.1 \%)$. The 
A
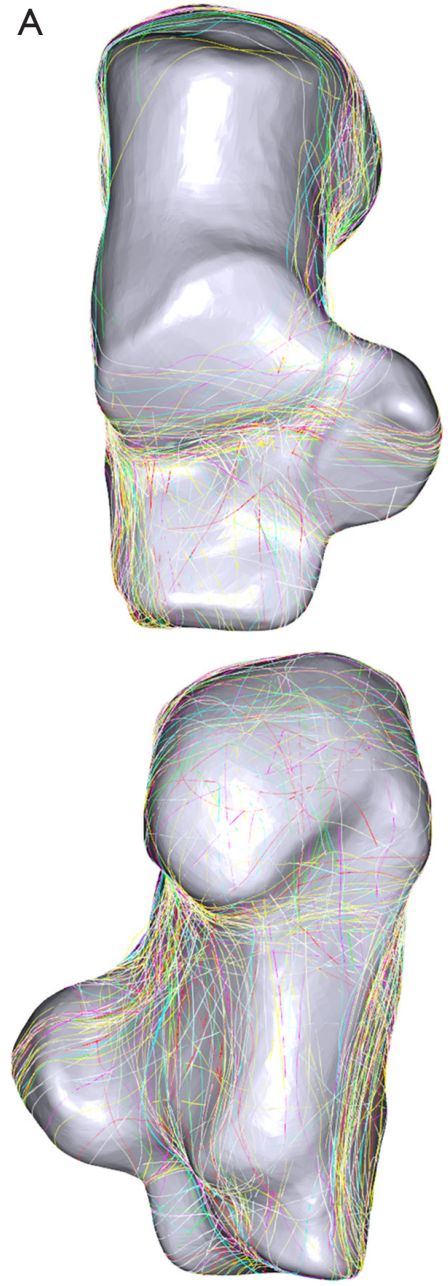

B
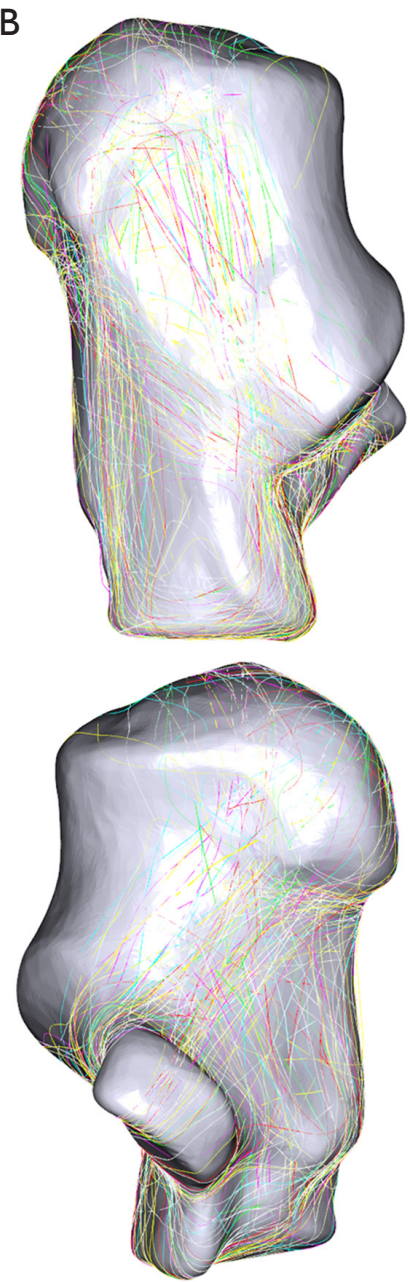

C
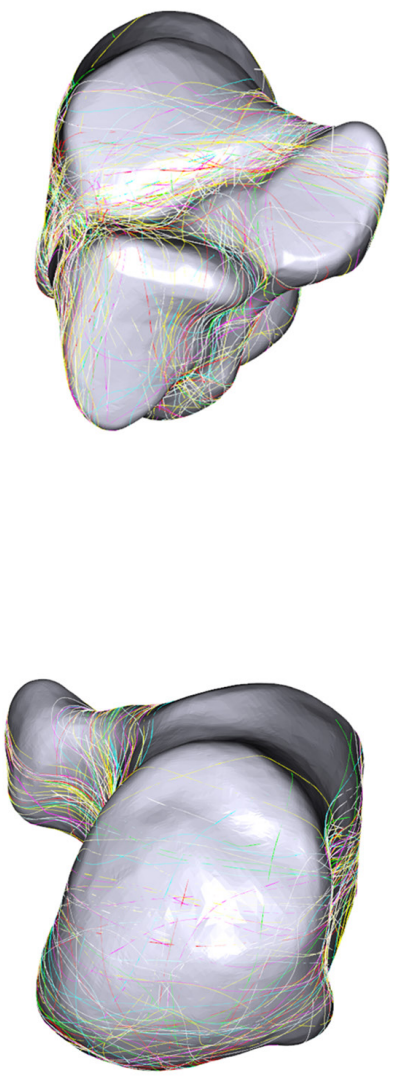

Figure 3 The fracture line distribution of Group A tongue-type calcaneal fractures was created using E-3D Medical 18.01 software. (A) Top and bottom perspectives; (B) lateral and medial perspectives; (C) front and rear perspectives.

Group C fracture line involved the anterior calcaneus and is represented as zone $\mathrm{A}(17,85.0 \%)$, zone $\mathrm{B}(5,25.0 \%)$, zone $\mathrm{C}(13,65.00 \%)$, and no involvement $(0,0.0 \%)$. There were no significant differences in the distribution of the $\mathrm{A}$, B, and C zones of the APC among the Group A, Group B, and Group C subtypes. The number of uninvolved APCs in Group A was significantly higher than those in Group B and Group C (Table 2).

In Group A, the most prevalent types were Type AC (24, 35.3\%) and Type A (11, 16.2\%). In Group B, the most common types were Type AC (20, 41.7\%), Type AB $(8,16.7 \%)$, and Type C $(8,16.7 \%)$. In Group C, the most prevalent types were Type AC $(8,40.0 \%)$ and Type A $(5,25.0 \%)$ (Table 2).

\section{Discussion}

In recent years, 3D CT reconstruction technology has been widely used in fracture diagnosis and classification, and is more intuitive and accurate than $\mathrm{X}$-ray diagnosis. However, whether the traditional fracture classification method can be improved by 3D CT reconstruction technology is worthy of further study. The emergence of fracture mapping technology visually demonstrated the morphological characteristics of the fracture line, and proposed new ideas for fracture diagnosis, classification, surgical plan and internal fixation instrument design. Fracture maps can be divided into two-dimensional (2D) and 3D maps; 2D maps can only analyze the orientation and distribution of fracture 

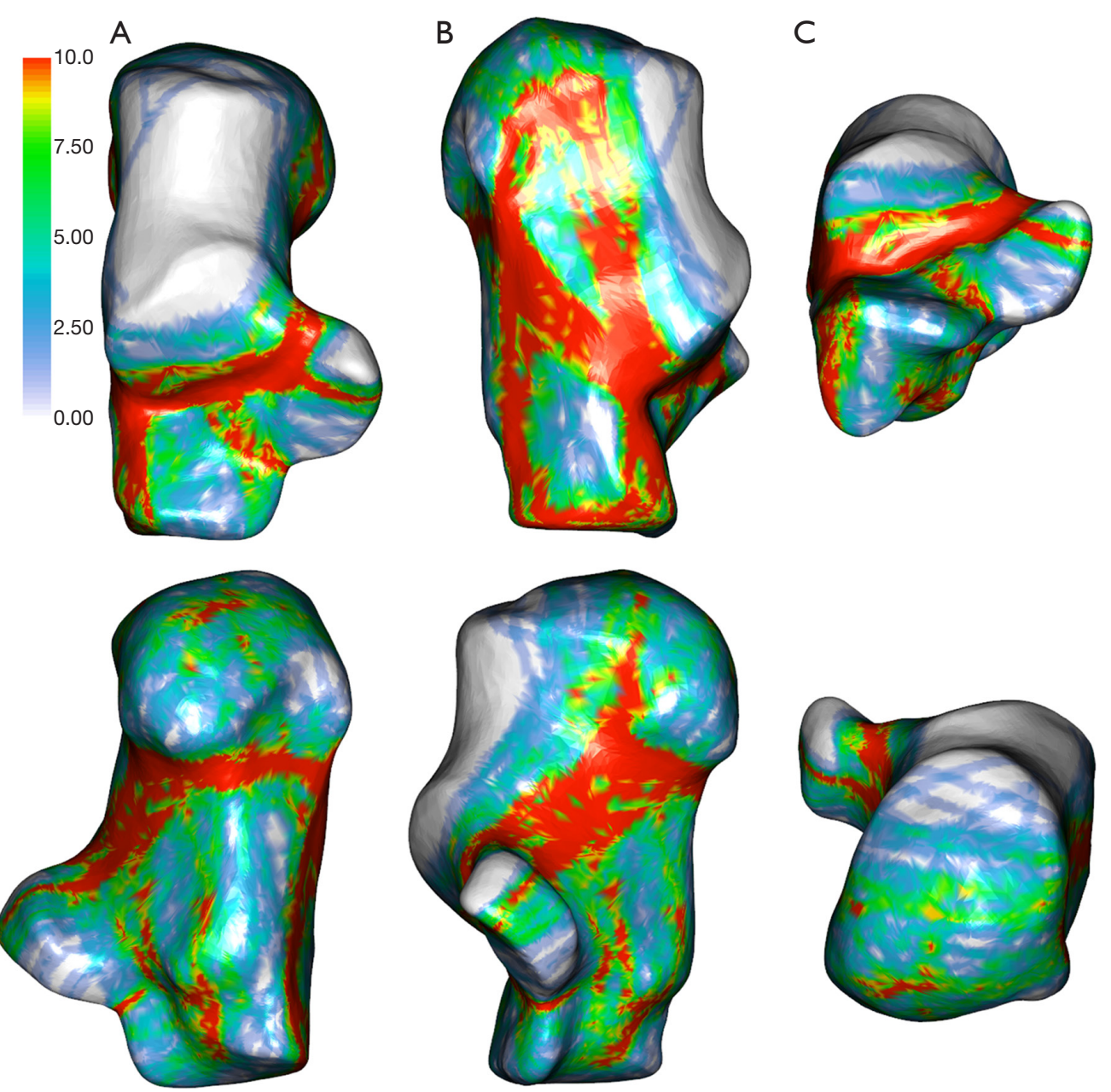

Figure 4 The fracture line heatmap distribution of Group A tongue-type calcaneal fractures was created using E-3D Medical 18.01 software. (A) Top and bottom perspectives; (B) lateral and medial perspectives; (C) front and rear perspectives.

lines on a certain section, while 3D maps can intuitively analyze the morphological characteristics of fractures in a 3D manner.

In 2009, Armitage et al. (8) superimposed the fracture lines of 90 cases of scapular fractures to form a fracture map, which was used to study the distribution of fracture lines. In 2013, Cole et al. (12) increased the superimposition of the defect area when studying the articular surface of pilon fractures. In 2015, Molenaars et al. (13) modified Cole et al.'s method to perform a virtual reduction of fracture fragments on CT axial images, and applied it to study the characteristics of tibial plateau fractures. In 2016, Mellema et al. (14-16) conducted research on the articular surface of the radial head, coronoid process, and olecranon, and further drew a heatmap that quantified the frequency of fractures.

In this process, research has also evolved from simply showing the distribution of fracture lines to heatmapping, which shows the frequency of fractures from a 2D drawing of the fracture line to CT tomographic reduction and then $3 \mathrm{D}$ reconstruction reduction. In this study, a $3 \mathrm{D}$ entity of calcaneal fracture was used for reduction and registration, and a $3 \mathrm{D}$ fracture line was subsequently drawn on the template to more intuitively and quantitatively generate a heatmap that displays the morphological characteristics of tongue-type calcaneal fractures and the internal fixation safety zone. This study provides a reference for the diagnosis and treatment of calcaneal fractures.

According to the description of Essex-Lopresti (5), when a person falls from a height, his ankle is perpendicular to 
A
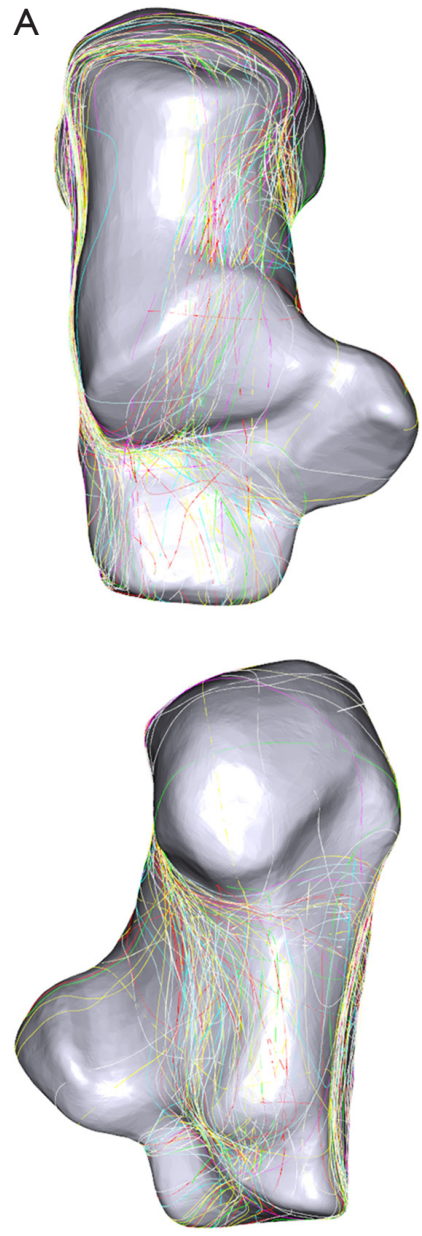

B
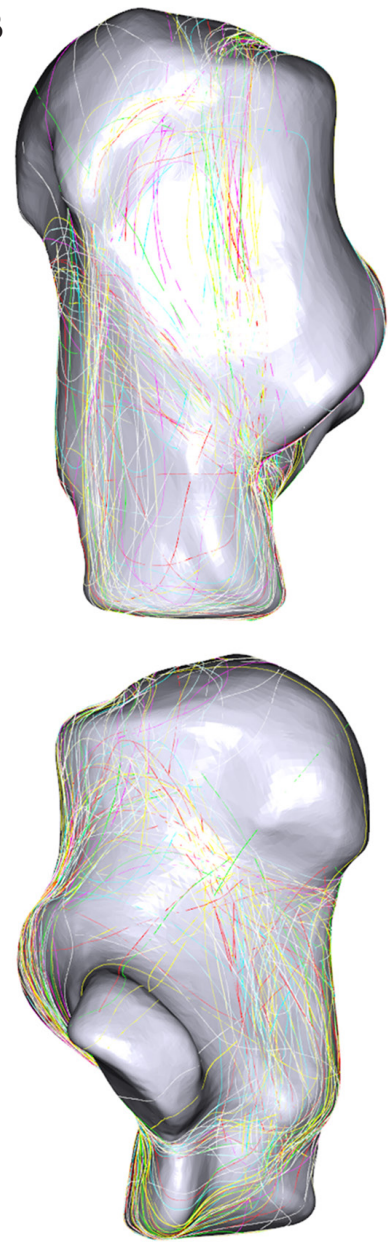

C
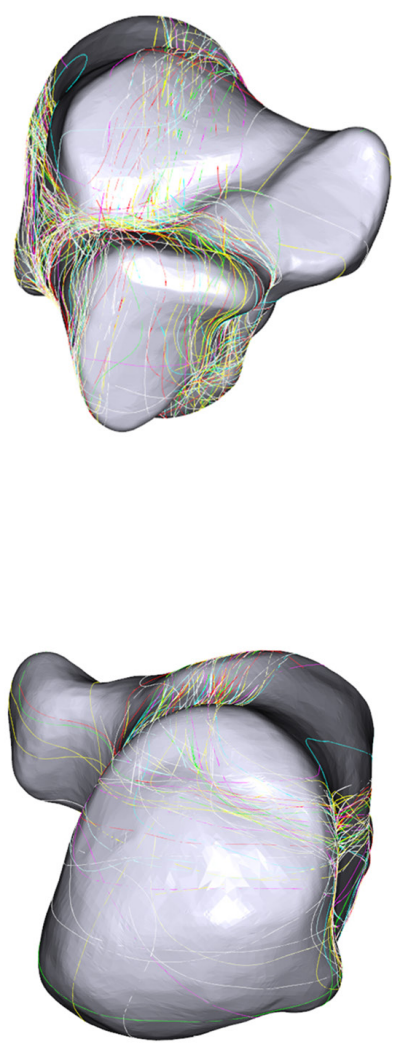

Figure 5 The fracture line distribution of Group B tongue-type calcaneal fractures was created using E-3D Medical 18.01 software. (A) Top and bottom perspectives; (B) lateral and medial perspectives; (C) front and rear perspectives.

the ground, and the axial load splits the posterior subtalar articular and the sustentaculum tali. When the force passes through the lateral approach, the posterior subtalar flips, the talus splits the lateral wall of the calcaneus, and the lateral surface produces an oblique fracture line starting from the angle of Gissane. When the force passes through medially, the sustentaculum tali is split, and the force continues, resulting in a secondary fracture line. The direction of the posterior branch of this line is variable, and horizontal extension to the calcaneal tuberosity produces the described "tongue-type fracture". In Group B, the primary and secondary fracture lines of the typical tongue-type calcaneal fracture are shown; the fracture line of the posterior subtalar articular surface was distributed longitudinally, and the concentrated area was the middle of the articular surface. This result is consistent with Essex-Lopresti. In
Group A, the fracture line of the posterior subtalar articular surface was distributed horizontally, and the concentrated area was the middle and lower subtalar articular surface, which suggests that Group A may be caused by axial pressure in the oblique posterior direction. Group $\mathrm{C}$ has the characteristics of both Group A and Group B; the posterior subtalar articular surface fracture lines were simultaneously distributed horizontally and vertically. The calcaneal body fracture line distribution was at the upper medial and lateral surfaces. We believe that axial pressure may first cause Group A fractures, which continue to progress posteriorly to cause Group C fractures. In this study, the subtypes of tongue-type calcaneal fractures were classified from a $3 \mathrm{D}$ perspective.

The sustentaculum tali is a special structure of the calcaneus that plays an important role in supporting 

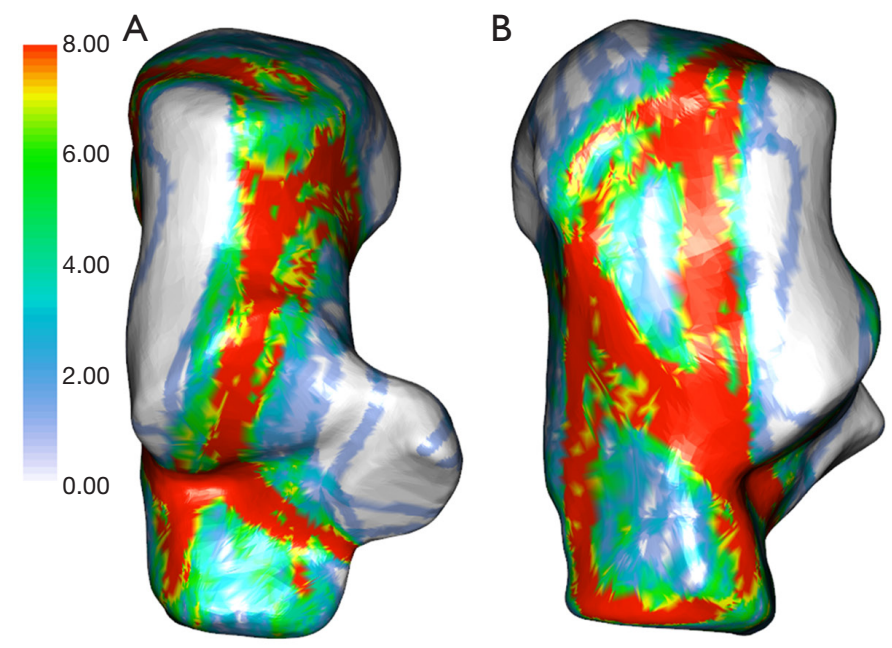

C
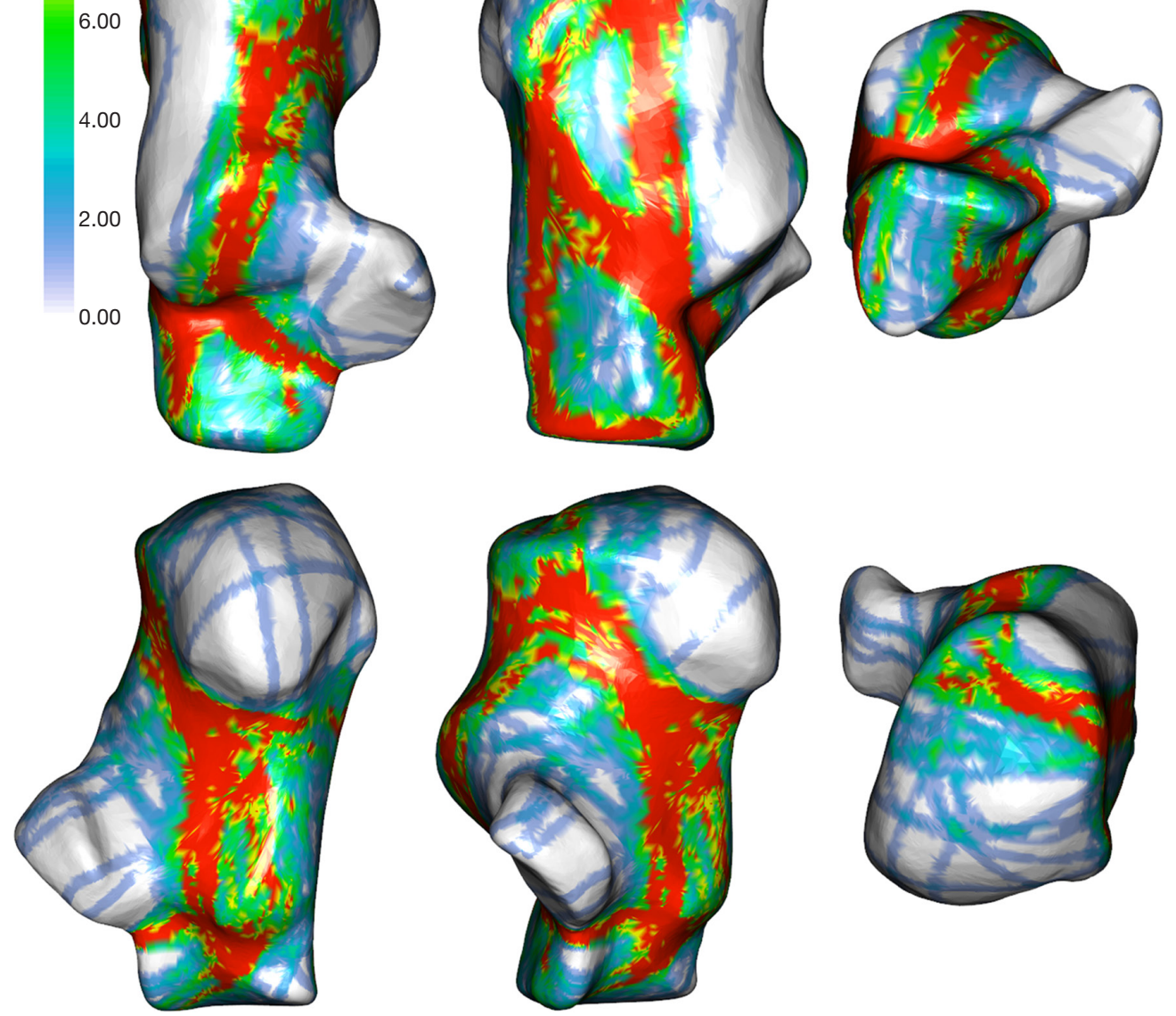

Figure 6 The fracture line heatmap distribution of Group B tongue-type calcaneal fractures was created using E-3D Medical 18.01 software. (A) Top and bottom perspectives; (B) lateral and medial perspectives; (C) front and rear perspectives.

body weight and transferring stress (17). Zwipp et al. (18) believed that reduction of the sustentaculum tali fragment is crucial for most patients with intra-articular calcaneal fractures. The present study showed that Group B fractures are caused by vertical violent splitting, and the longitudinal fracture line was located at the base of the sustentaculum tali. The Group A fracture contained the sustentaculum tali as a transverse fracture along the middle subtalar joint, and the fracture line continued the transverse fracture line of the posterior subtalar joint. Therefore, for Group B fractures, multiple screws need to be inserted from the lateral side of the calcaneus to the medial side to fix the sustentaculum tali fracture. For Group A fractures, it is sufficient to firmly fix the tongue fragment.

Calcanocuboid joint injuries are more common clinically, and the incidence of calcaneal fractures is $33-76 \%(17,19-20)$. However, most scholars only emphasize the importance of anatomical reduction of the posterior subtalar articular surface of the calcaneus and the hindfoot, while ignoring the impact of fracture and dislocation of the calcaneocubic joint on foot function. In Groups A, B, and C, the concentrated area of the fracture line in the APC was the lateral wall (zone A) and the transition area between the anterior calcaneus and the middle subtalar joint (zone C). The head of the calcaneal longitudinal screw was stable in the middle of the calcaneocuboid joint (zone B).

At present, conventional 3D CT reconstruction of the calcaneus cannot display the subtalar joint and the calcaneocuboid joint because the calcaneus is covered by the talus and cuboid. The calcaneus entity can be separated 
A
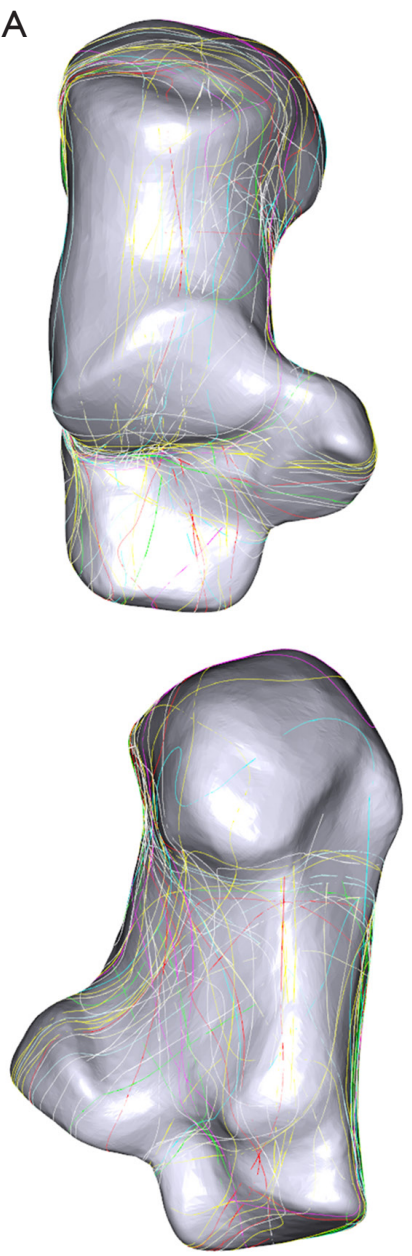

B
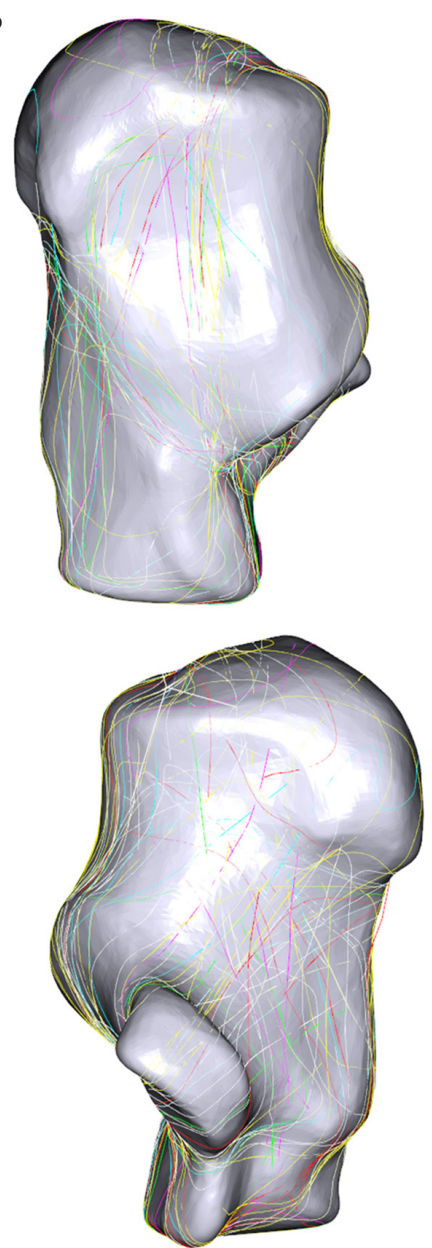

C
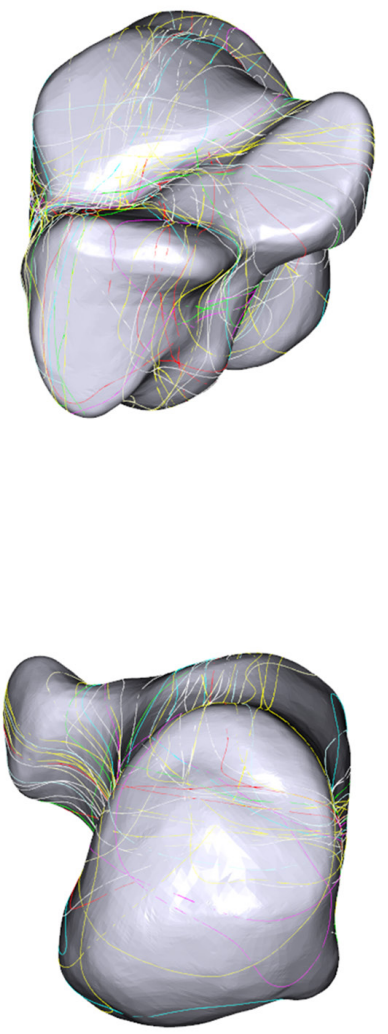

Figure 7 The fracture line distribution of Group C tongue-type calcaneal fractures was created using E-3D Medical 18.01 software. (A) Top and bottom perspectives; (B) lateral and medial perspectives; (C) front and rear perspectives.

from the other ankle bones to draw a fracture map through the split function of Mimics Research 20.0 software. Through this research, doctor can indirectly assess the distribution of the subtalar joint fracture line of tonguetype calcaneal fracture via the fracture line involved the calcaneal body. This study can guide the reduction strategy of calcaneal fractures and the direction of screw placement. The map of tongue-type calcaneal fracture can reflect the most concentrated and sparse area of the fracture line. The internal fixation design of the calcaneus can refer to the fracture map, place its tip and tail in the sparse area, and the body passes through the dense area of fracture lines. In this way, the stability of fracture fixation is improved, and the possibility of internal fixation failure is reduced. Numerous biomechanical and finite element analyses have discussed the internal fixation methods of calcaneal fractures; however, there is no standard calcaneal fracture model (21-26). In this study, the area with the highest fracture line weight was clarified, and thus, the results of this research can be used as a standard for laboratory modeling in future biomechanics and finite element analysis research.

With the development of intraoperative X-ray fluoroscopic equipment and internal fixation devices, the use of percutaneous screws for fixation of calcaneal fractures has achieved better results (27-29). This study found that the sparse areas of Group A fracture lines are the upper-posterior side of the calcaneal body, the anterior part of the middle subtalar joint, and the B area of the APC. The tails of the two longitudinal screws should then be distributed parallel to the upper part of the calcaneal tuberosity, and their heads should be distributed vertically in area B of the APC. The tip of screw should be placed 

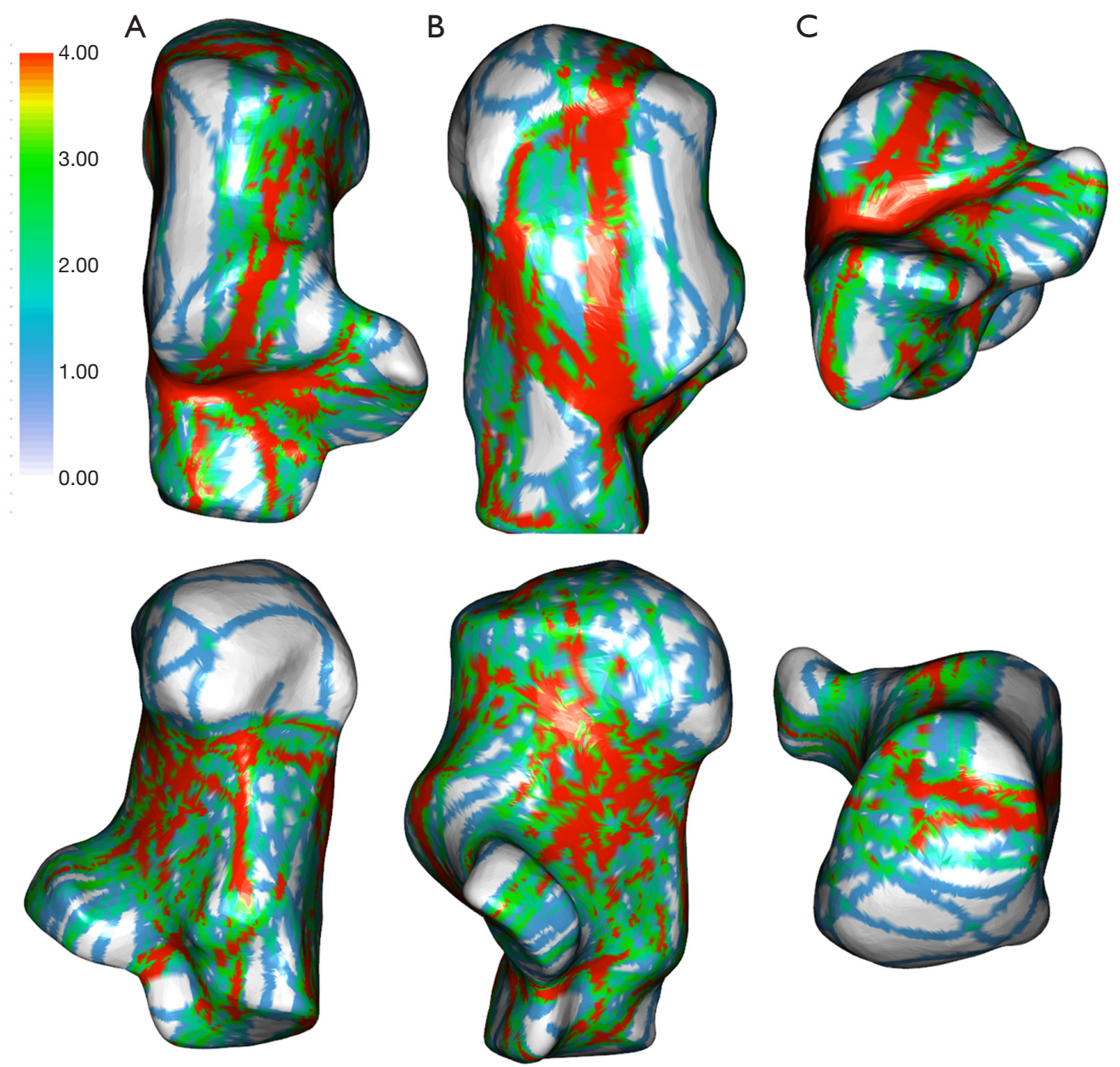

Figure 8 The fracture line heatmap distribution of Group C tongue-type calcaneal fractures was created using E-3D Medical 18.01 software. (A) Top and bottom perspectives; (B) lateral and medial perspectives; (C) front and rear perspectives.

in the front part of the sustentaculum tail, and only one screw available (Figure 9). For Group B fractures, the sparse areas of the fracture line are the upper-lateral sides of the calcaneal body, the lower part of the calcaneal tuberosity, the middle subtalar joint, and area B of the APC. The tails of the two longitudinal screws should be located on the upper-lateral and lower sides of the calcaneal tuberosity, and the heads should be located in zone B of the APC. Since the sustentaculum tail is intact, two screws can be placed in parallel from calcaneal lateral surface to fix the sustentaculum tail (Figure 10). For Group C fractures, the only areas where the fracture line is sparse are the upper part of the calcaneus and area B of the APC. Therefore, it is necessary to use a steel plate to fix the fracture fragments, and at the same time, a longitudinal screw whose tail is located on the upper-lateral side of the calcaneal tuberosity and head is located in area B of the APC should be added (Figure 11).

In the era of big data, the fracture map uses visualization methods to clearly depict the fracture site and shape of the fracture line, which is helpful for the design of surgical approaches and the selection of internal fixation devices. However, fracture maps have shortcomings. Firstly, due to anatomical variation, some of the $3 \mathrm{D}$ reconstruction images cannot perfectly match the standard template, and the depicted fracture line deviates from the actual fracture line. Secondly, this study only supplements the previous classification methods of calcaneal fractures and does not create a new classification method. Thirdly, the current grouping method is not related to the long-term clinical effect, and further improvements are needed in future research. 
Table 2 Tongue-type calcaneal fracture line distribution in the APC $(n=136)$

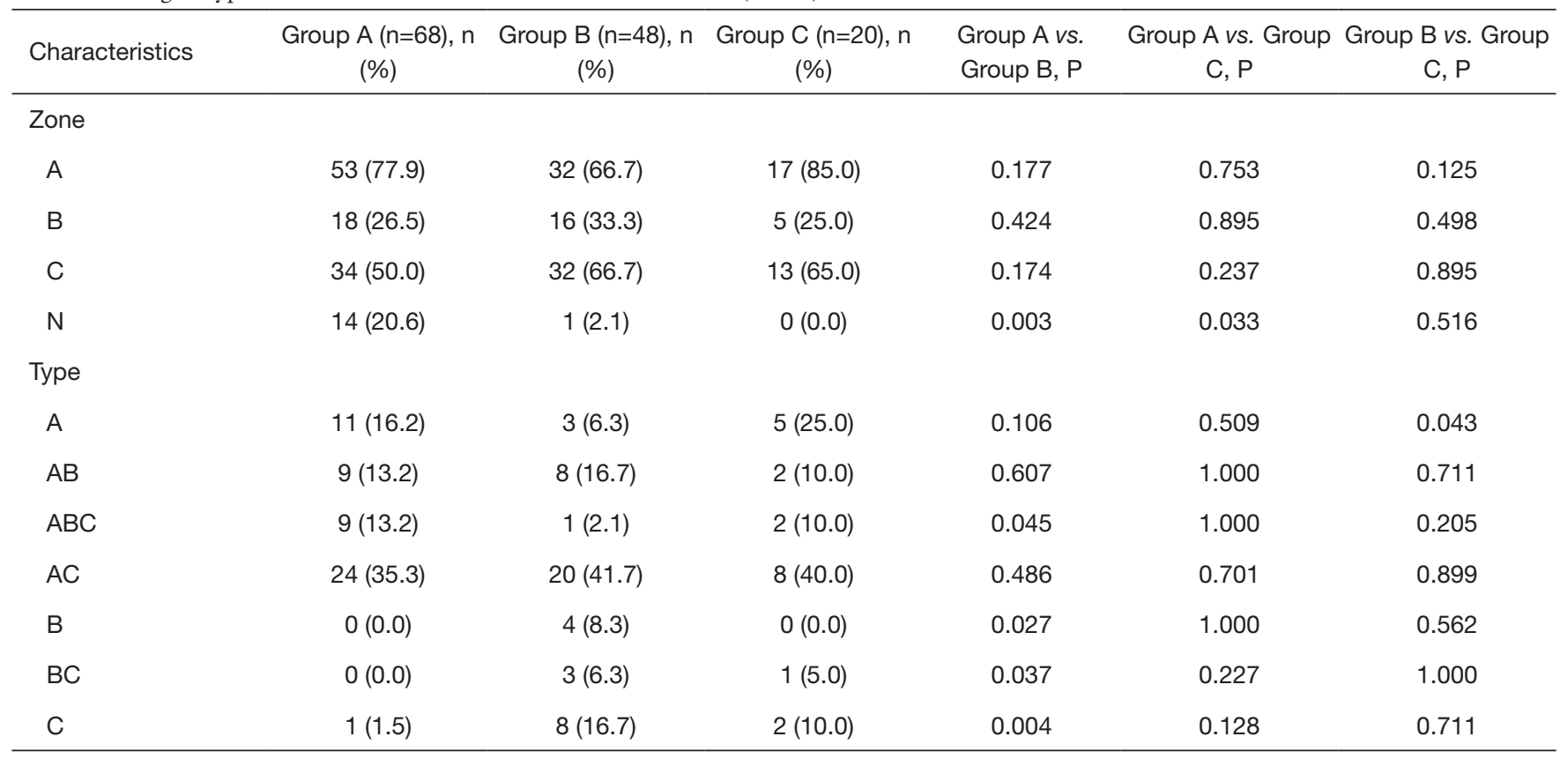

APC, anterior part of the calcaneus.

A

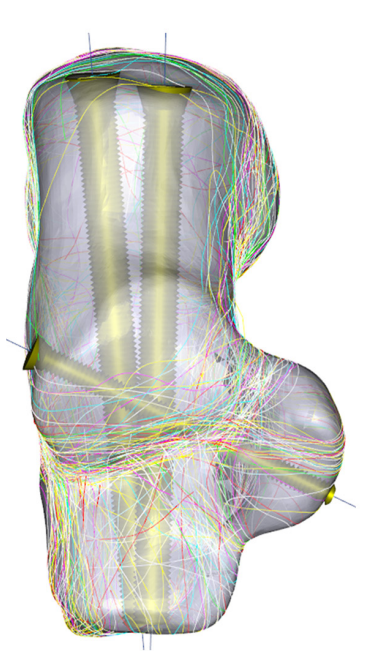

B

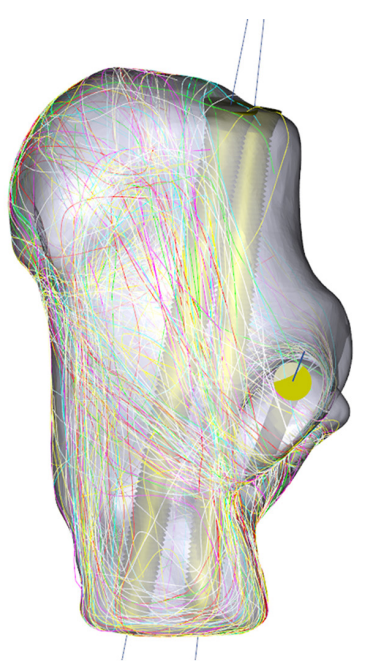

C

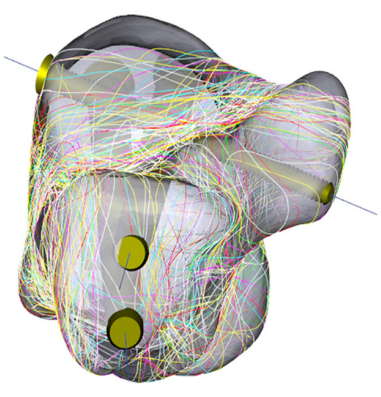

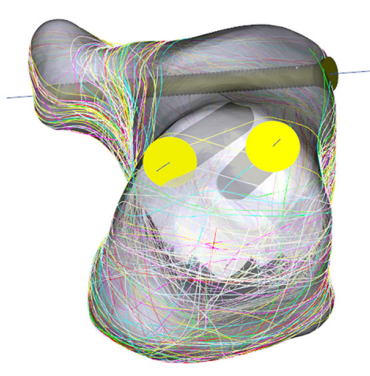

Figure 9 Optimal screw distribution in Group A tongue-type calcaneal fractures was created using E-3D Medical 18.01 software. (A) Top perspective; (B) lateral perspective; (C) front perspective; and (D) rear perspective.

\section{Conclusions}

This study investigates the distribution characteristics of fracture lines in subgroups of tongue-type calcaneal fractures for the first time. The results can help doctors improve their understanding of tongue-type calcaneal fractures, optimize internal fixation design, and provide a standard model for biomechanical experiments. 
A

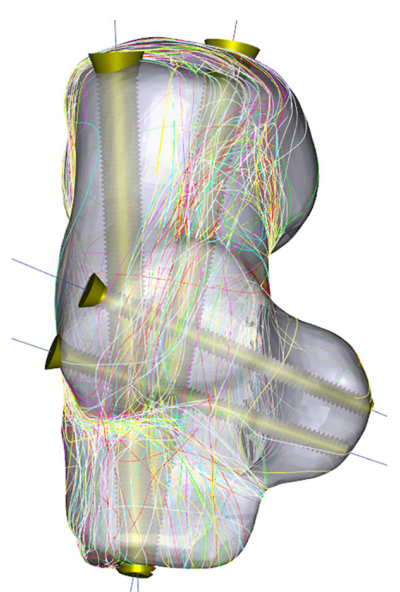

B

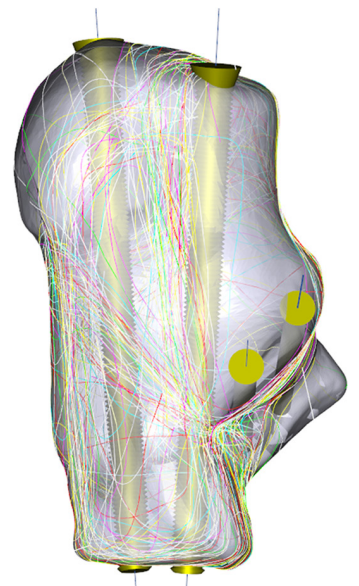

C

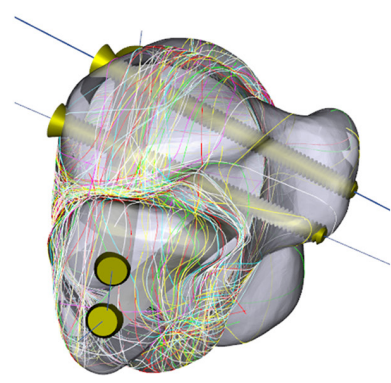

D

Figure 10 Optimal screw location in Group B tongue-type calcaneal fractures was created using E-3D Medical 18.01 software. (A) Top perspective; (B) lateral perspective; (C) front perspective; and (D) rear perspective.

A

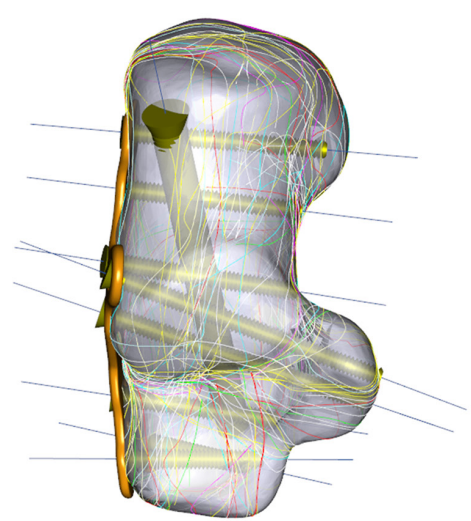

B

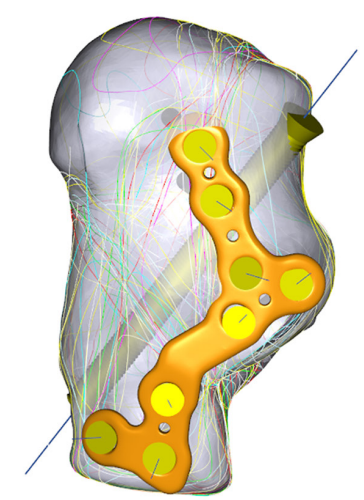

C

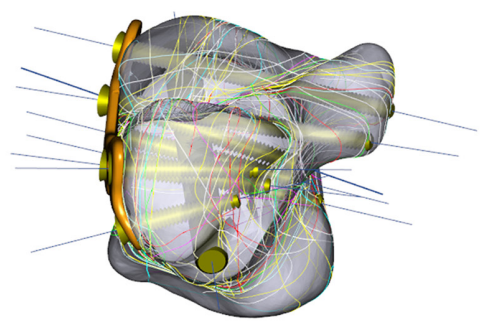

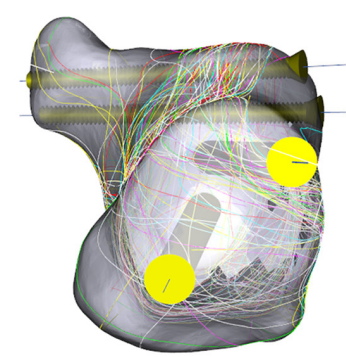

Figure 11 Optimal plate and screw location in Group C tongue-type calcaneal fractures was created using E-3D Medical 18.01 software. (A) Top perspective; (B) lateral perspective; (C) front perspective; and (D) medial perspective.

\section{Acknowledgments}

Funding: None.

\section{Footnote}

Reporting Checklist: The authors have completed the MDAR reporting checklist. Available at https://dx.doi. org/10.21037/atm-21-6168
Data Sharing Statement: Available at https://dx.doi. org/10.21037/atm-21-6168

Conflicts of Interest: All authors have completed the ICMJE uniform disclosure form (available at https://dx.doi. org/10.21037/atm-21-6168). The authors have no conflicts of interest to declare.

Ethical Statement: The authors are accountable for all 
aspects of the work in ensuring that questions related to the accuracy or integrity of any part of the work are appropriately investigated and resolved. All procedures performed in this study involving human participants were in accordance with the Declaration of Helsinki (as revised in 2013). The study was approved by institutional ethics board of Jincheng General Hospital (No. 20200901) and informed consent was taken from all the patients.

Open Access Statement: This is an Open Access article distributed in accordance with the Creative Commons Attribution-NonCommercial-NoDerivs 4.0 International License (CC BY-NC-ND 4.0), which permits the noncommercial replication and distribution of the article with the strict proviso that no changes or edits are made and the original work is properly cited (including links to both the formal publication through the relevant DOI and the license). See: https://creativecommons.org/licenses/by-nc-nd/4.0/.

\section{References}

1. Bernstein J, Ahn J. In brief: Fractures in brief: calcaneal fractures. Clin Orthop Relat Res 2010;468:3432-4.

2. Potter MQ, Nunley JA. Long-term functional outcomes after operative treatment for intra-articular fractures of the calcaneus. J Bone Joint Surg Am 2009;91:1854-60.

3. Yoshimura I, Ichimura R, Kanazawa K, et al. Simultaneous use of lateral calcaneal ostectomy and subtalar arthroscopic debridement for residual pain after a calcaneal fracture. J Foot Ankle Surg 2015;54:37-40.

4. Vicenti G, Solarino G, Caizzi G, et al. Balloon-assisted reduction, pin fixation and tricalcium phosphate augmentation for calcaneal fracture: A retrospective analysis of 42 patients. Injury 2018;49 Suppl 3:S94-9.

5. ESSEX-LOPRESTI P. The mechanism, reduction technique, and results in fractures of the os calcis. Br J Surg 1952;39:395-419.

6. Howells NR, Hughes AW, Jackson M, et al. Interobserver and intraobserver reliability assessment of calcaneal fracture classification systems. J Foot Ankle Surg 2014;53:47-51.

7. Sanders R, Fortin P, DiPasquale T, et al. Operative treatment in 120 displaced intraarticular calcaneal fractures. Results using a prognostic computed tomography scan classification. Clin Orthop Relat Res 1993;(290):87-95.

8. Armitage BM, Wijdicks CA, Tarkin IS, et al. Mapping of scapular fractures with three-dimensional computed tomography. J Bone Joint Surg Am 2009;91:2222-8.
9. Wang C, Zhu Y, Long H, et al. Three-dimensional mapping of distal humerus fracture. J Orthop Surg Res 2021;16:545.

10. Zhan Y, Zhang Y, Xie X, et al. Three-dimensional fracture mapping of multi-fragmentary patella fractures (AO/OTA 34C3). Ann Transl Med 2021;9:1364.

11. Guo X, Liang X, Jin J, et al. Evaluation of Sanders Type 2 Joint Depression Calcaneal Fractures in 197 Patients from a Single Center Using Three-Dimensional Mapping. Med Sci Monit 2021;27:e932748.

12. Cole PA, Mehrle RK, Bhandari M, et al. The pilon map: fracture lines and comminution zones in OTA/AO type 43C3 pilon fractures. J Orthop Trauma 2013;27:e152-6.

13. Molenaars RJ, Mellema JJ, Doornberg JN, et al. Tibial Plateau Fracture Characteristics: Computed Tomography Mapping of Lateral, Medial, and Bicondylar Fractures. J Bone Joint Surg Am 2015;97:1512-20.

14. Mellema JJ, Eygendaal D, van Dijk CN, et al. Fracture mapping of displaced partial articular fractures of the radial head. J Shoulder Elbow Surg 2016;25:1509-16.

15. Mellema JJ, Doornberg JN, Dyer GS, et al. Distribution of coronoid fracture lines by specific patterns of traumatic elbow instability. J Hand Surg Am 2014;39:2041-6.

16. Lubberts B, Mellema JJ, Janssen SJ, et al. Fracture line distribution of olecranon fractures. Arch Orthop Trauma Surg 2017;137:37-42.

17. Badillo K, Pacheco JA, Padua SO, et al. Multidetector CT evaluation of calcaneal fractures. Radiographics 2011;31:81-92.

18. Zwipp H, Tscherne H, Thermann H, et al. Osteosynthesis of displaced intraarticular fractures of the calcaneus. Results in 123 cases. Clin Orthop Relat Res 1993;(290):76-86.

19. Gallino RM, Gray AC, Buckley RE. The outcome of displaced intra-articular calcaneal fractures that involve the calcaneocuboid joint. Injury 2009;40:146-9.

20. Stapleton JJ, Kolodenker G, Zgonis T. Internal and external fixation approaches to the surgical management of calcaneal fractures. Clin Podiatr Med Surg 2010;27:381-92.

21. Xu C, Liu H, Li M, et al. A Three-Dimensional Finite Element Analysis of Displaced Intra-Articular Calcaneal Fractures. J Foot Ankle Surg 2017;56:319-26.

22. Ouyang H, Deng Y, Xie P, et al. Biomechanical comparison of conventional and optimised locking plates for the fixation of intraarticular calcaneal fractures: a finite element analysis. Comput Methods Biomech Biomed Engin 2017;20:1339-49.

23. Ni M, Wong DW, Niu W, et al. Biomechanical comparison of modified Calcanail system with plating 
fixation in intra-articular calcaneal fracture: A finite element analysis. Med Eng Phys 2019;70:55-61.

24. Ramlee MH, Gan HS, Daud SA, et al. Stress Distributions and Micromovement of Fragment Bone of Pilon Fracture Treated With External Fixator: A Finite Element Analysis. J Foot Ankle Surg 2020;59:664-72.

25. Pan M, Chai L, Xue F, et al. Comparisons of external fixator combined with limited internal fixation and open reduction and internal fixation for Sanders type 2 calcaneal fractures: Finite element analysis and clinical outcome. Bone Joint Res 2017;6:433-8.

26. Chen CH, Hung C, Hsu YC, et al. Biomechanical evaluation of reconstruction plates with locking, nonlocking, and hybrid screws configurations in calcaneal fracture: a finite element model study. Med Biol Eng Comput 2017;55:1799-807.

Cite this article as: Guo X, Liang X, Jin J, Chen J, Liu J, Qiao Y, Cheng J, Zhao J. Three-dimensional computed tomography mapping of 136 tongue-type calcaneal fractures from a single centre. Ann Transl Med 2021;9(24):1787. doi: 10.21037/atm$21-6168$
27. Li M, Lian X, Yang W, et al. Percutaneous Reduction and Hollow Screw Fixation Versus Open Reduction and Internal Fixation for Treating Displaced Intra-Articular Calcaneal Fractures. Med Sci Monit 2020;26:e926833.

28. Jin C, Weng D, Yang W, et al. Minimally invasive percutaneous osteosynthesis versus ORIF for Sanders type II and III calcaneal fractures: a prospective, randomized intervention trial. J Orthop Surg Res 2017;12:10.

29. Sampath Kumar V, Marimuthu K, Subramani S, et al. Prospective randomized trial comparing open reduction and internal fixation with minimally invasive reduction and percutaneous fixation in managing displaced intra-articular calcaneal fractures. Int Orthop 2014;38:2505-12.

(English Language Editor: A. Kassem) 